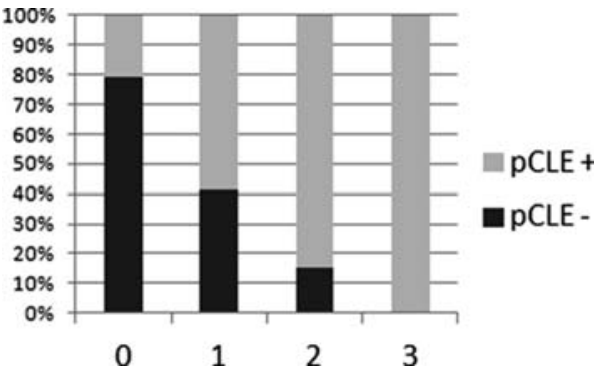

Abstract OC-017 Figure 1 Correlation between pCLE and biomarkers. X-axis: number of positive biomarkers

AFI+ areas, followed by random biopsies as per Seattle protocol. pCLE sequences were graded according to published criteria. Cyclin A and p53 expression were assessed by immunohistochemistry and aneuploidy by flow-cytometry on AFI-targeted biopsies. Statistical analyses were performed using chi-square test.

Results AFI-targeted pCLE correctly classified all the HGD/EC patients and had a sensitivity and specificity for any grade of dysplasia of 93 and $83 \%$, respectively. The Seattle protocol had similar sensitivity for HGD/IMC and any grade of dysplasia (83 and $89 \%$, respectively). For the per-location analysis, a total of 155 endoscopic areas were analysed with pCLE and molecular biomarkers. pCLE had a sensitivity and a specificity for HGD/ IMC and any grade of dysplasia of $100 / 64 \%$ and $78 / 75 \%$, respectively. Overall, $40 \%$ of pCLE irregular sequences corresponded to non-dysplastic areas (false positive). We found a statistically significant enrichment $(\mathrm{p}<0.001)$ of the three molecular biomarkers in pCLE irregular areas (Figure 1). After exclusion of dysplastic areas, a significant correlation between pCLE irregularity and biomarker positivity was retained $(\mathrm{p}=$ 0.008). The presence of at least 1 positive biomarker significantly correlated with dysplasia both in pCLE irregular $(\mathrm{p}=$ $0.01)$ and $\mathrm{pCLE}$ regular areas $(\mathrm{p}=0.05)$.

Conclusion AFI-targeted pCLE has a high diagnostic accuracy for dysplasia in BO. Tissue biomarkers are a useful adjunct to characterise the field of molecular abnormality associated with optical dysplasia. These results suggest that the presence of pCLE irregularity, even in the absence of histological dysplasia, relates to molecular changes and may warrant close follow up. Disclosure of Interest None Declared.

\section{OC-018 GASTRIN INCREASES MIR-222 EXPRESSION IN GASTRIC EPITHELIAL CELLS IN VITRO AND HYPERGASTRINAEMIC INS-GAS MICE IN VIVO}

${ }^{1} \mathrm{~K}$ Lloyd*, ${ }^{1} \mathrm{~A}$ O'Hara, ${ }^{2} \mathrm{~A}$ Varro, ${ }^{1} \mathrm{DM}$ Pritchard. ' Gastroenterology, University of Liverpool, Liverpool, UK; ${ }^{2}$ Physiology, University of Liverpool, Liverpool, UK

\subsection{6/gutjnl-2014-307263.18}

Introduction Gastric adenocarcinoma occurs in some patients who are infected with Helicobacter pylori. Gastrin is a cofactor in gastric carcinogenesis and elevated serum concentrations are found in the preneoplastic condition atrophic gastritis. MicroRNAs (miRNAs) are small non-coding RNAs that post transcriptionally regulate numerous mRNAs and play critical roles in cell physiology. Previous studies have suggested that H.pylori infection dysregulates miRNAs to control gastric inflammation, cell cycle progression, apoptosis and cell survival. We hypothesised that gastrin would also induce alterations in gastric miRNAs and that these may influence cancer development.
Methods Human gastric adenocarcinoma cells that have been stably transfected with the human CCK2 receptor $\left(\mathrm{AGS}_{\mathrm{GR}}\right)$ were treated with $0.1-100 \mathrm{nM}$ gastrin for 2-48 h. Small RNAs were isolated and reverse transcribed using the Qiagen miScript PCR system kit. miRNA expression profiling was determined by qPCR using miScript PCR arrays (in triplicate) and further validated using miRNA primer assays (in quadruplicate). Cycle passing threshold (Ct) was normalised to RNU62 expression and miRNA relative expression calculated using $\Delta \Delta \mathrm{C}_{\mathrm{T}}$ method. miR-222 levels were measured in gastric mucosal scrapings from 10 week old male and female ( $n=3$ per group) wild-type FVB/ $\mathrm{N}$ mice and transgenic hypergastrinaemic INS-GAS mice on the same genetic background. Comparisons were made using unpaired t-tests with Bonferroni correction, $\mathrm{P}<0.05$ was considered significant.

Results miR-376c and miR-222 were significantly overexpressed in gastrin treated $\mathrm{AGS}_{\mathrm{GR}}$ cells, by 5.2-fold $[\mathrm{p}<0.01]$ and 2.3fold $[\mathrm{p}<0.0001]$ respectively. However only the increase in miR-222 expression was confirmed using qPCR. Maximal increased expression of miR-222 (9-fold $[\mathrm{p}<0.01]$ ) was seen after $10 \mathrm{nM}$ G17 treatment for $24 \mathrm{~h}$ in serum free media. Increased miR-222 expression was completely reversed by pretreatment with the CCK-2 receptor antagonist YM022 $(100 \mathrm{nM})$. miR-222 expression was also significantly increased in 10 week old female and male INS-GAS mice, compared with FVB/N mice (by 5.3-fold and 2.3-fold respectively).

Conclusion Gastrin induces gastric miRNA alterations, specifically miR-222 overexpression, both in vitro and in vivo. This was fully reversed by pre-treatment with YM022 in vitro. Since miR-222 overexpression has previously been linked to decreased expression of tumour suppressor proteins such as $\mathrm{p} 27^{\mathrm{Kip} 1}$ and increased oncogenesis, these data support the hypothesis that elevated gastrin may induce pathological changes via disruption of miRNA (particularly miR-222) expression. Further studies are needed to determine the mechanisms by which gastrin-induced miR-222 overexpression affects gastric pathology.

Disclosure of Interest K. Lloyd Grant/research support from: Trio Medicines Ltd, A. O'Hara: None Declared, A. Varro Grant/ research support from: Trio Medicines Ltd, D. Pritchard Grant/ research support from: Trio Medicines Ltd.

\section{OC-019 OPTIMISING THE PERFORMANCE OF MAGNETIC ASSISTED CAPSULE ENDOSCOPY (MACE) OF THE UPPER GI TRACT USING CT MODELLING}

I Rahman*, M Kay, T Bryant, S Pelitari, BD Dimitrov, P Patel. University Hospital Southampton, Southampton, UK

\subsection{6/gutjnl-2014-307263.19}

Introduction Capsule endoscopy, employed to investigate the small bowel, is now being further developed to visualise the upper GI tract. In a pig model, using a hand held magnet, we have demonstrated that magnetic assisted capsule endoscopy (MACE) in the stomach is feasible. However, it is unclear what the best methodology is to achieve complete gastric luminal views in humans. Our aim was to utilise CT modelling of the abdomen to determine the optimal placements of a capsule endoscope in the stomach to allow complete mucosal visualisation and to determine the optimal placement of the hand held magnet to aid pyloric traversing.

Methods Using multiplanar reformatting, 100 good quality contrast abdominal CT scans were analysed to assess luminal visualisation by a magnetic capsule endoscope from 5 fixed stations throughout the stomach. From each station, we assessed the 